\title{
Non-Fourier heat conduction study for steady states in metallic nanofilms
}

\author{
WANG HaiDong ${ }^{1}$, LIU JinHui ${ }^{1}$, GUO ZengYuan ${ }^{1 *} \&$ TAKAHASHI Koji ${ }^{2}$ \\ ${ }^{1}$ Key Laboratory for Thermal Science and Power Engineering of Ministry of Education, School of Aerospace, Tsinghua University, Beijing \\ 100084, China, \\ ${ }^{2}$ Graduate School of Engineering, Kyushu University, Fukuoka 819-0395, Japan
}

Received February 22, 2012; accepted May 2, 2012

\begin{abstract}
As a fundamental theory of heat transfer, Fourier's law is valid for most traditional conditions. Research interest in non-Fourier heat conditions is mainly focused on heat wave phenomena in non-steady states. Recently, the thermomass theory posited that, for steady states, non-Fourier heat conduction behavior could also be observed under ultra-high heat flux conditions at low ambient temperatures. Significantly, this is due to thermomass inertia. We report on heat conduction in metallic nanofilms from large currents at low temperatures; heat fluxes of more than $1 \times 10^{10} \mathrm{~W} \mathrm{~m}^{-2}$ were used. The measured average temperature of the nanofilm is larger than that based on Fourier's law, with temperature differences increasing as heat flux increased and ambient temperature decreased. Experimental results for different film samples at different ambient temperatures reveal that non-Fourier behavior exists in metallic nanofilms in agreement with predictions from thermomass theory.
\end{abstract}

Fourier's law, non-Fourier behavior, thermomass theory, metallic nanofilm

Citation: Wang H D, Liu J H, Guo Z Y, et al. Non-Fourier heat conduction study for steady states in metallic nanofilms. Chin Sci Bull, 2012, 57: 3239-3243, doi: $10.1007 / \mathrm{s} 11434-012-5288-7$

Early in 1822, the French mathematical physicist, Joseph Fourier studied many experimental results on heat conduction summarized in his famous Fourier's law, advancing a linear relationship between heat flux and temperature gradient [1]. Subsequently, Fourier's law has been proved valid in numerous engineering application fields. Because the governing equation of Fourier's law is parabolic, it leads to infinite propagation speeds for thermal disturbances, which opposes basic physical principles. To resolve this problem, many researchers developed some modification of Fourier's law. Cattaneo [2], Vernotte [3], and Morse and Feshbach [4] subsequently developed a thermal wave model by introducing a relaxation time for heat flux:

$$
\begin{gathered}
\tau_{0} \frac{\partial q}{\partial t}+q=-\lambda \frac{\partial T}{\partial x} \\
\tau_{0} \frac{\partial^{2} T}{\partial t^{2}}+\frac{\partial T}{\partial t}=\alpha \frac{\partial^{2} T}{\partial x^{2}}
\end{gathered}
$$

\footnotetext{
*Corresponding author (email: demgzy@ tsinghua.edu.cn)
}

where $\tau_{0}, \lambda$, and $\alpha$ are relaxation time, thermal conductivity, and thermal diffusivity, respectively. The model is commonly called the C-V model. From eq. (1), heat is seen to propagate with finite speed, quite distinct from Fourier's law. Such behavior is referred to as non-Fourier heat conduction. Later, Tzou [5] proposed a dual phase model involving two kinds of relaxation times: heat flux and temperature. The dual phase model and governing equation are expressed as follows:

$$
\begin{gathered}
q\left(x, t+\tau_{q}\right)=-\lambda \frac{\partial T}{\partial x}\left(x, t+\tau_{T}\right), \\
\tau_{q} \frac{\partial^{2} T}{\partial t^{2}}+\frac{\partial T}{\partial t}=\alpha \frac{\partial^{2} T}{\partial x^{2}}+\tau_{T} \alpha \frac{\partial^{3} T}{\partial x^{2} \partial t},
\end{gathered}
$$

where $\tau_{q}$ and $\tau_{T}$ are the respective heat flux and temperature relaxation times. Eq. (4) describes different heat conduction behaviors from heat diffusion, electron- phonon coupling to thermal waves by applying the appropriate $\tau_{q}$ and $\tau_{T}$.

The non-Fourier models mentioned above have one thing in common; namely each reduces to Fourier's law for 
steady states. In another words, it is known so far that non-Fourier heat conduction occurs only in non-steady states. Recently, Guo and co-workers [6-8] proposed the notion of thermomass based on Einstein's mass-energy relation; thermomass is defined as the equivalent mass of thermal energy. Heat conduction is understood as the flow of thermomass and Newtonian mechanics can be used to analyze its motion:

$$
\begin{gathered}
\tau_{\mathrm{h}} \frac{\partial q}{\partial t}-\frac{\lambda_{\mathrm{I}} q}{2 \gamma \rho C^{2} T^{2}} \frac{\partial T}{\partial t}+\frac{\lambda_{\mathrm{I}} q}{2 \gamma \rho^{2} C^{3} T^{2}} \frac{\partial q}{\partial x} \\
+\lambda_{\mathrm{I}}\left(1-\frac{q^{2}}{2 \gamma \rho^{2} C^{3} T^{3}}\right) \frac{\partial T}{\partial x}+q=0 \\
\tau_{\mathrm{h}} \frac{\partial^{2} T}{\partial t^{2}}+\frac{\partial T}{\partial t}=\alpha\left(1-\frac{q^{2}}{2 \gamma \rho^{2} C^{3} T^{3}}\right) \frac{\partial^{2} T}{\partial x^{2}}-\frac{2 \tau_{\mathrm{h}} q}{\rho C T} \frac{\partial^{2} T}{\partial t \partial x},
\end{gathered}
$$

where $\tau_{\mathrm{h}}, \rho, C, q, \lambda_{I}$ and $\gamma_{\mathrm{h}}$ are the respective characteristic time, material density, specific heat, heat flux, intrinsic thermal conductivity, and Grüneisen parameter. Eq. (5) is the momentum conservation equation of thermomass and eq. (6) is the general heat conduction equation based on eq. (5). Eq. (6) is a hyperbolic equation which can be used to describe thermal wave phenomena. If one ignores thermomass inertia, eq. (6) reduces to Fourier's law. Furthermore, here reported for the first time, the thermomass theory predicts the existence of non-Fourier heat conduction in steady states. The steady expression of eq. (6) in one dimension is:

$$
\begin{gathered}
\lambda_{\mathrm{I}}\left(1-\frac{q^{2}}{2 \gamma_{\mathrm{h}} \rho^{2} C^{3} T^{3}}\right) \frac{\mathrm{d} T}{\mathrm{~d} x}+q=0, \\
\lambda_{\mathrm{A}}=\lambda_{\mathrm{I}}\left(1-\frac{q^{2}}{2 \gamma_{h} \rho^{2} C^{3} T^{3}}\right),
\end{gathered}
$$

where $\lambda_{\mathrm{A}}$ is the apparent thermal conductivity connected with $\lambda_{\mathrm{I}}$. Eq. (7) states that heat conduction processes will differ from Fourier's law under high heat flux and low temperature conditions. As heat flux increases and temperature decreases, differences between eq. (7) and Fourier's law widens.

The available experimental studies of non-Fourier heat conduction have only focused on thermal wave phenomena at very low temperatures $[9,10]$ or under ultra-short pulsed-laser heating conditions [11]. For example, Chester [12] and Narayana et al. [13] measured the propagation speed of solid thermal waves, initially referred to as second-sound in liquid helium. Brorson et al. [14] measured the propagation speed of thermal disturbances in metallic nanofilms using a femtosecond laser thermo-reflectance technique and found speeds close to electron Fermi velocities. For this paper, we studied heat conduction in metallic nanofilms heated by large currents at low temperatures. The steady non-Fourier heat conduction predicted by eq. (7) was confirmed.

\section{Experimental setup}

According to eq. (7), the steady non-Fourier heat conduction occurs only under ultra-low temperature, ultra-high heat flux conditions. A liquid helium cooling system was used to provide a minimum ambient temperature of $2.8 \mathrm{~K}$. Metallic nanofilms can sustain large heating currents with maximum heat fluxes of more than $10^{10} \mathrm{~W} \mathrm{~m}^{-2}$. Co-workers in Kyushu university of Japan prepared high-qualified $\mathrm{Au}$ nanofilms using electron-beam physical vapor deposition. The SEM pictures of Au nanofilms are shown in Figure 1. The geometric parameters of prepared $\mathrm{Au}$ nanofilms are listed in Table 1.

To avoid heat loss into the substrate, nanofilms are suspended from the Si substrate, as seen in Figure 1. A two-stage vacuum pumping system is used to maintain the air pressure below $10^{-4} \mathrm{~Pa}$ around the nanofilms, and thus eliminate heat convection. A thermal radiation shield is

Table 1 Geometric parameters of Au nanofilms

\begin{tabular}{cccc}
\hline Film No. & Thickness $(\mathrm{nm})$ & Width $(\mathrm{nm})$ & Length $(\mu \mathrm{m})$ \\
\hline A & 76 & 302 & 10.10 \\
B & 76 & 308 & 9.59 \\
\hline
\end{tabular}

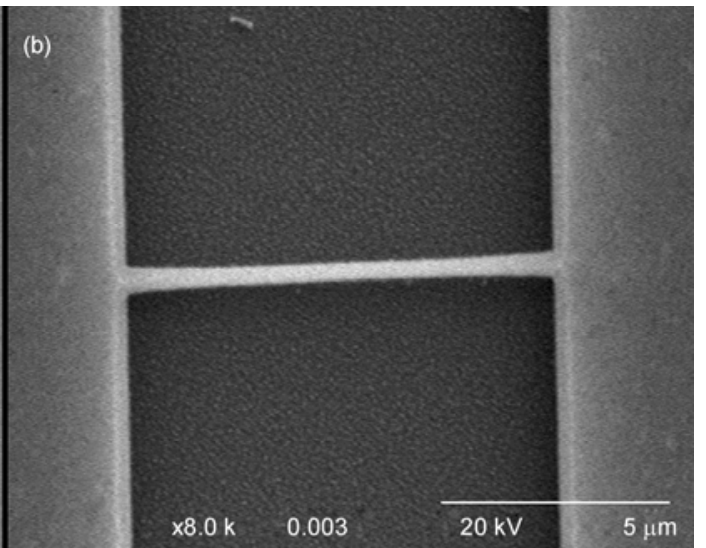

Figure 1 SEM pictures of suspended Au nanofilms. (a) Image from side view; (b) image from top view. 
fashioned to isolate the device from ambient radiation. An ITC503 temperature controller from Oxford Instruments guarantees temperature monitoring to within a $0.1 \mathrm{~K}$ resolution. Two high-precision digital voltmeters (Keitheley 2002) and a DC power supply (Advantest R6243) are used to measure the resistance of $\mathrm{Au}$ nanofilm using a four-probe method.

\section{Direct current heating method}

\subsection{One dimensional heat conduction model}

Figure 2 is a schematic of one-dimensional heat conduction used to describe direct current heating. Each Au nanofilm is suspended between two heat sinks used as electrodes. A parabolic temperature distribution forms along the Au nanofilm from the DC current heating. The temperature predicted by thermomass theory is slightly higher than that predicted by Fourier's law; the significant difference is seen to arise from a steady non-Fourier heat conduction source caused by the spatial thermomass inertia. Measurements of local temperature in nanofilms are extremely difficult. Here, we measure the average temperature of the nanofilm using the linear relationship between resistance and temperature of metals, the Au nanofilm being treated here as both electric heater and thermometer. Under large current heating conditions, the average temperature of the Au nanofilm is measured and compared with the temperature predicted by Fourier's law to verify the existence of a non-Fourier component.

Although eq. (7) is actually derived for dielectrics, in metals, electrons are the main energy carriers and the state equation of the thermomass of electrons is different from that of phonons. While their thermomass state equations differ, the existence of non-Fourier heat conduction under ultra-high heat flux conditions remains unchanged; this will be proved later in section 3 .

\subsection{Measurement of electronic and thermal conductiv- ities of Au nanofilms}

To estimate the temperature distribution of $\mathrm{Au}$ nanofilm accurately, the electronic and thermal conductivities need to be measured first. The conductivities are greatly reduced from the bulk values due to significant size effects in

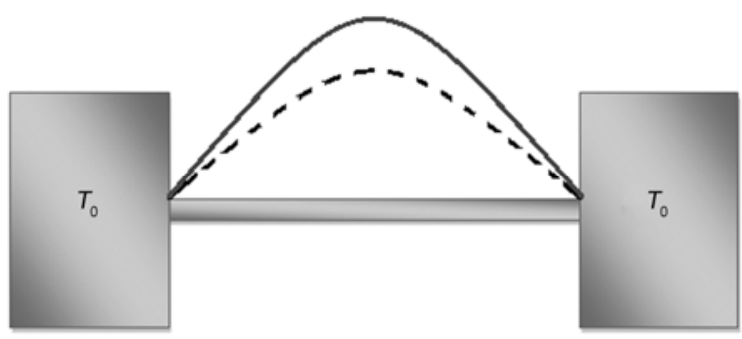

Figure 2 Schematic diagram of direct current heating method. nanofilms. Here, the direct-current heating method is used to measure the electrical and thermal conductivities simultaneously. The heating power during the measurement is only several microwatts to ensure the maximum temperature rise is below $10 \mathrm{~K}$. The thermomass inertia is then negligible and Fourier's law holds valid. The parabolic temperature distribution of one-dimensional heat conduction can be expressed as $[15,16]$ :

$$
T(x)=T_{0}+\frac{I U}{2 w \delta \lambda_{\mathrm{f}}} x-\frac{I U}{2 w \delta l \lambda_{\mathrm{f}}} x^{2},
$$

where $x=0$ refers to the left end of the nanofilm; $U$ and $I$ are the voltage and current; $l, w$ and $\delta$ are the respective length, width, and thickness of the nanofilm; and $\lambda_{\mathrm{f}}$ is the nanofilm thermal conductivity. The average temperature of the nanofilm can be derived from eq. (9):

$$
\Delta T=\frac{I U l}{12 w \delta \lambda_{\mathrm{f}}} .
$$

The resistance-temperature linear relationship of metals is [17]:

$$
R_{\mathrm{f}}=R_{0}+\beta_{\mathrm{f}} R_{\mathrm{r}} \Delta T=R_{0}+\frac{\beta_{\mathrm{f}} R_{\mathrm{r}}}{\lambda_{\mathrm{f}}} \frac{I U l}{12 w \delta},
$$

where $R_{\mathrm{f}}$ is the film resistance, $R_{0}$ and $R_{\mathrm{r}}$ are the respective resistances at ambient temperature $T_{0}$ and reference temperature $T_{\mathrm{r}}$, and $\beta_{\mathrm{f}}$ is the temperature coefficient of resistance, which is given as

$$
\beta_{\mathrm{f}}=\frac{R_{0}-R_{\mathrm{r}}}{R_{\mathrm{r}}\left(T_{0}-T_{\mathrm{r}}\right)}
$$

Thus, for the thermal conductivity of nanofilm, we have

$$
\lambda_{\mathrm{f}}=\frac{\beta_{\mathrm{f}} R_{\mathrm{r}}}{b} \frac{l}{12 w \delta},
$$

where $b$ is the coefficient of proportionality between resistance and heating power. Meanwhile, the electrical conductivity $\sigma_{\mathrm{f}}$ is

$$
\sigma_{\mathrm{f}}=\frac{l}{R_{0} w \delta} .
$$

The uncertainty in the measured thermal conductivity is estimated as

$$
\frac{\Delta \lambda_{\mathrm{f}}}{\lambda_{\mathrm{f}}} \approx \sqrt{3\left(\frac{\Delta R}{R}\right)^{2}+\left(\frac{\Delta l}{l}\right)^{2}+\left(\frac{\Delta T_{0}}{T_{0}}\right)^{2}+\left(\frac{\Delta b}{b}\right)^{2}+\left(\frac{\Delta w}{w}\right)^{2}+\left(\frac{\Delta \delta}{\delta}\right)^{2}} .
$$

Here, the voltage uncertainty is $\Delta U / U=\Delta I / I \leqslant 0.01 \%$; the temperature uncertainty $\Delta T_{0} / T_{0} \leqslant 0.01 \%$; the uncertainty in geometric measurements $\Delta l / l=\Delta w / w \leqslant 1 \%$; the uncertainty in film thickness $\Delta \delta / \delta \leqslant 0.1 \%$; and the uncertainty from linear fitting $\Delta b / b \leqslant 0.1 \%$. Consequently, the overall uncertainty in estimating the thermal conductivity is $\Delta \lambda / \lambda \leqslant 2 \%$, and the corresponding temperature uncertainty is below $\pm 3 \mathrm{~K}$.

The uncertainty in the temperature coefficient of resistance is: 


$$
\frac{\Delta \beta_{\mathrm{f}}}{\beta_{\mathrm{f}}} \approx \sqrt{4\left(\frac{\Delta R}{R}\right)^{2}+\left(\frac{\Delta T_{0}}{T_{0}}\right)^{2}} .
$$

In considering all parameters involved, we obtain $\Delta \beta_{\mathrm{f}} / \beta_{\mathrm{f}}$ $\leqslant 0.11 \%$.

\subsection{Measurement by large current heating}

During the experiment, the two film samples A and B are measured continuously over a range of ambient temperatures and heating powers. The measured temperatures are then compared with predictions from Fourier's law. Based on the thermomass theory, the thermomass inertia is negligible under low heating power conditions and Fourier's law holds. As heating power increases, the heat flux in the nanofilm increases and thermomass inertia will be more substantial. The measured temperature should be higher than that predicted by Fourier's law, which would demonstrate the existence of a non-Fourier heat conduction component.

\section{Experimental results}

The measured electrical and thermal conductivities of nanofilm samples A and B are shown in Figure 3.

The measured conductivities of samples A and B show great consistency, demonstrating the high accuracy and repeatability of the experiment. We remark that each film sample had been vacuum annealed before measurement taking. Annealing reduces the lattice defects of the film and conductivities will rise correspondingly.

The experimental results for large current heating are plotted in Figures 4 and 5.

Figures 4 and 5 graph average temperature vs. heating power curves for samples A and B at different ambient temperatures; circles represent the measured temperature, squares represent the temperature predicted by Fourier's law, $w$ is the film width and $T_{0}$ is the ambient temperature. The insets of Figures 4 and 5 are enlargements at maximum temperatures. With the maximum heat flux of Au nanofilm

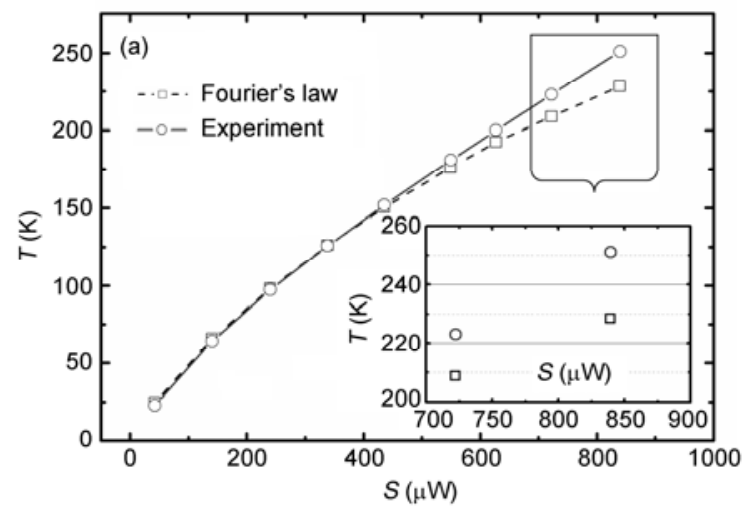

at more than $10^{10} \mathrm{~W} \mathrm{~m}^{-2}$, we note non-negligible temperature rises at both ends of the film. Using the measured thermal conductivity as a given parameter, the temperature rise at these ends can be accurately calculated by a $3 \mathrm{D}$ heat conduction model that has already been accounted for in Fourier's law. For a detailed analysis, the experimental parameters and measured results are listed in Table 2.

In Table $2, T_{0}, W_{\max }, q_{\max }, T_{\max -\mathrm{E}}, T_{\max -\mathrm{F}}$, and $\Delta T_{\max }$ are the respective ambient temperature, max-heating power, maxheat flux, max-measured temperature, max-temperature predicted by Fourier's law, and max-temperature difference. Based on the measured results, it is concluded that:

(1) When the heating power is low, thermomass inertia is negligible and Fourier's law holds. As heat flux increases, thermomass inertia becomes substantial, leading to a significant non-Fourier heat conduction component.

(2) Repeated measurements on samples A and B demonstrate that non-Fourier heat conduction could be seen under low temperature, high heat flux conditions. $\Delta T_{\max }$, marked with asterisks in Table 2, are the measured values at the same heat flux level. It is noted that $\Delta T_{\max }$ increases as temperature decreases. This trend accords well with thermomass theory.

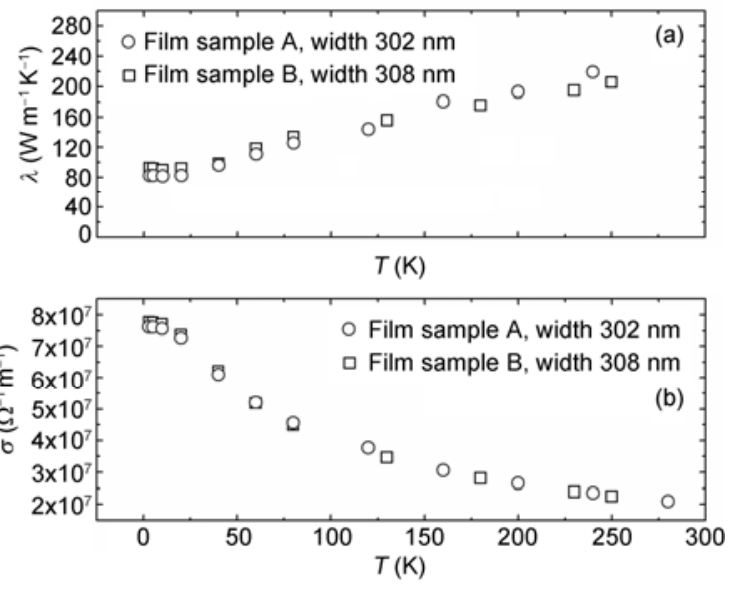

Figure 3 Measured electrical and thermal conductivities of samples A and B. (a) Thermal conductivity; (b) electrical conductivity.

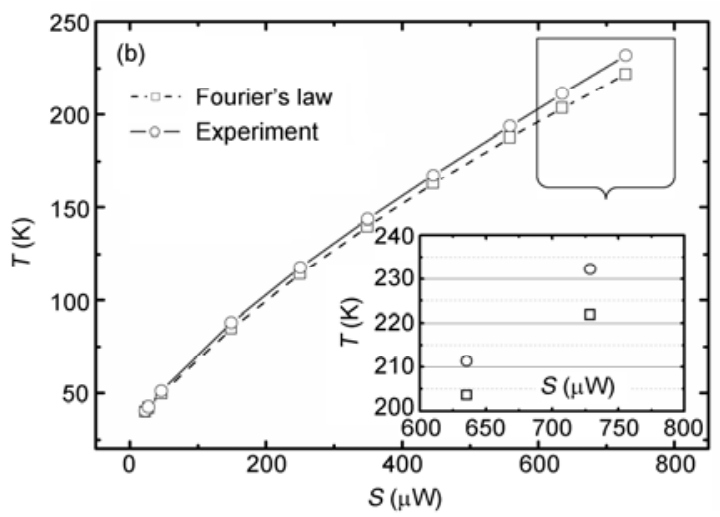

Figure 4 Experimental results for sample A at different ambient temperatures. (a) Ambient temperature $3 \mathrm{~K}$; (b) ambient temperature $30 \mathrm{~K}$. 

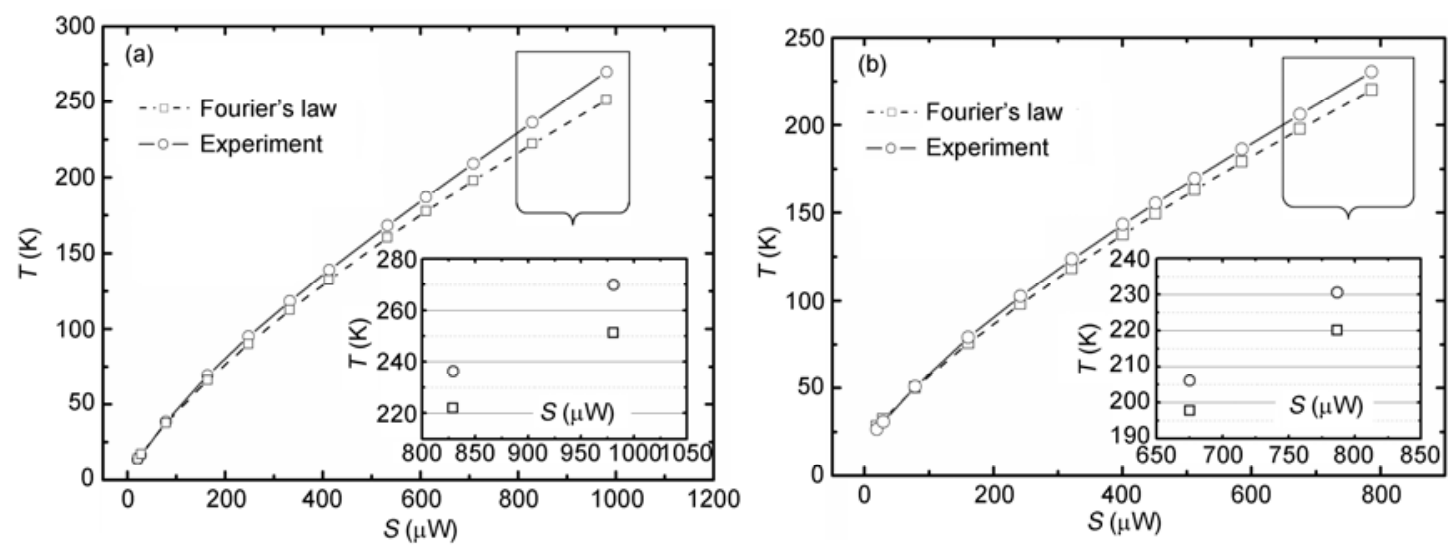

Figure 5 Experimental results for sample B at different ambient temperatures. (a) Ambient temperature $5 \mathrm{~K}$; (b) ambient temperature $20 \mathrm{~K}$.

Table 2 Experimental results under maximum current heating conditions

\begin{tabular}{|c|c|c|c|c|c|c|}
\hline No. & $T_{0}(\mathrm{~K})$ & $W_{\max }(\mu \mathrm{W})$ & $q_{\max }\left(\mathrm{Wm}^{-2}\right)$ & $T_{\max -\mathrm{E}}(\mathrm{K})$ & $T_{\max -\mathrm{F}}(\mathrm{K})$ & $\Delta T_{\max }(\mathrm{K})$ \\
\hline \multirow{2}{*}{ A } & 3.0 & 839.457 & $1.83 \times 10^{10}$ & 251.098 & 228.399 & 22.699 \\
\hline & 3.0 & 722.412 & $1.57 \times 10^{10}$ & 223.097 & 208.758 & $14.339^{*}$ \\
\hline A & 30.0 & 728.758 & $1.59 \times 10^{10}$ & 232.222 & 221.803 & $10.419^{*}$ \\
\hline \multirow{2}{*}{ B } & 5.0 & 980.864 & $2.10 \times 10^{10}$ & 269.830 & 251.255 & 18.575 \\
\hline & 5.0 & 829.274 & $1.77 \times 10^{10}$ & 236.224 & 221.971 & $14.253^{*}$ \\
\hline $\mathrm{B}$ & 20.0 & 786.732 & $1.68 \times 10^{10}$ & 230.525 & 220.042 & $10.483^{*}$ \\
\hline
\end{tabular}

* Measured values at the same heat flux level.

\section{Concluding remarks}

(1) Past non-Fourier heat conduction studies focused on transient thermal wave phenomena. The thermomass theory based on Einstein's mass-energy relation posits that steady non-Fourier heat conduction exists under low temperature, high heat flux conditions.

(2) The electrical and thermal conductivities of Au nanofilms have been measured simultaneously using a direct current heating method. The measured results of the different film samples show great consistency.

(3) The measurement by large current heating at low temperatures has been repeated in two different nanofilms. The measured average temperature is higher than that predicted by Fourier's law, indicating the existence of steady non-Fourier heat conduction. Increases in $\Delta T_{\max }$ as heat flux increases and temperature decreases were shown to agree with the prediction of thermomass theory.

This work was supported by the National Natural Science Foundation of China (51076080, 51136001, 50730006) and the Tsinghua University Initiative Scientific Research Program.

1 Fourier J. Analytical Theory of Heat. New York: Dover Publishers, 1955. 84-86

2 Cattaneo C. Sulla conduzione del calore. Atti Sem Mat Fis Univ Modena, 1948, 3: 83-101

3 Vernotte P C R. Les paradoxes de la théorie continue de l'équation de la chaleur. Acad Sci, 1958, 246: 3154-3155

4 Morse P M, Feshbach H. Methods of Theoretical Physics. New York: McGraw-Hill, 1953. 165-166

5 Tzou D Y. The generalized lagging response in small-scale and high-rate heating. Int J Heat Mass Transfer, 1995, 38: 3231-3240

6 Wang H D, Cao B Y, Guo Z Y. Heat flow choking in carbon nanotubes. Int J Heat Mass Transfer, 2010, 53: 1796-1800

7 Cao B Y, Guo Z Y. Equation of motion of a phonon gas and non-Fourier heat conduction. J Appl Phys, 2007, 102: 053503

8 Guo Z Y, Cao B Y, Zhu H Y, et al. State equation of phonon gas and conservation equations for phonon gas motion(in Chinese). Acta Phys Sin, 2007, 56: 3306-3312

9 Peshkov V. "Second sound" in helium II. J Physics-USSR, 1944, 8: 381-381

10 Landau L. Theory of the superfluidity of helium II. Phys Rev, 1941, 60: 356-358

11 Wang H D, Ma W G, Zhang X, et al. Measurement of the thermal wave in metal films using femtosecond laser thermoreflectance system (in Chinese). Acta Phys Sin, 2010, 59: 3856-3862

12 Chester M. Second sound in solids. Phys Rev, 1963, 131: 2013-2015

13 Narayana V, Dynes R C. Observation of second sound in bismuth. Phys Rev Lett, 1972, 28: 1461-1465

14 Brorson S D, Fujimoto J G, Ippen E P. Femtosecond electronic heat-transport dynamics in thin gold-films. Phys Rev Lett, 1987, 59: 1962-1965

15 Wang H D, Liu J H, Zhang X, et al. Experimental study on the influences of grain boundary scattering on the charge and heat transport in gold and platinum nanofilms. Heat Mass Transfer, 2011, 47: 893-898

16 Zhang Q G, Cao B Y, Zhang X, et al. Size effects on the thermal conductivity of polycrystalline platinum nanofilms. J Phys: Condens Matter, 2006, 18: 7937-7950

17 Ma W G, Wang H D, Zhang X, et al. Different effects of grain boundary scattering on charge and heat transport in polycrystalline platinum and gold nanofilms. Chin Phys B, 2009, 18: 2035-2040

Open Access This article is distributed under the terms of the Creative Commons Attribution License which permits any use, distribution, and reproduction in any medium, provided the original author(s) and source are credited. 